\title{
Optimal Use of Perampanel in Asian Patients with Epilepsy: Expert Opinion
}

\author{
Yotin Chinvarun' \\ Chin-Wei Huang ${ }^{2}$ \\ Ye $\mathrm{Wu}^{3}$ \\ Hsiu-Fen Lee ${ }^{4}$ \\ Surachai Likasitwattanakul ${ }^{5}$ \\ Jing Ding ${ }^{6}$ \\ Takamichi Yamamoto $^{7}$ \\ 'Department of Neurology, \\ Phramongkutklao Royal Army Hospital \\ and Medical College, Bangkok, Thailand; \\ ${ }^{2}$ Department of Neurology, National \\ Cheng Kung University Hospital College \\ of Medicine, National Cheng Kung \\ University, Tainan, Taiwan; ${ }^{3}$ Department \\ of Pediatrics, Peking University First \\ Hospital, Beijing, People's Republic of \\ China; ${ }^{4}$ Division of Pediatric Neurology, \\ Children's Medical Center, Taichung \\ Veterans General Hospital, Taichung, \\ Taiwan; ${ }^{5}$ Division of Neurology, \\ Department of Pediatrics, Faculty of \\ Medicine, Siriraj Hospital, Mahidol \\ University, Bangkok, Thailand; \\ ${ }^{6}$ Department of Neurology, Zhongshan \\ Hospital, Fudan University, Shanghai, \\ People's Republic of China; ${ }^{7}$ Department \\ of Neurosurgery, Comprehensive \\ Epilepsy Center, Seirei Hamamatsu \\ General Hospital, Hamamatsu, Japan
}

Correspondence: Takamichi Yamamoto Department of Neurosurgery,

Comprehensive Epilepsy Center, Seirei

Hamamatsu General Hospital, 2-12-12

Sumiyoshi, Hamamatsu, Shizuoka, 430-

8558, Japan

Tel +8I 534742222

Fax +8I 534743094

Email taka-yamamd@sis.seirei.or.jp

\begin{abstract}
Perampanel is a once-daily, first-in-class AMPA receptor antagonist approved for the treatment of epilepsy and exhibits broad-spectrum efficacy in a range of seizure types when used as both monotherapy and adjunctive therapy. Clinical studies and real-world evidence have demonstrated the advantages of initiating perampanel at low doses and utilizing a slow titration strategy. Initiating perampanel at an early stage has also been shown to be associated with better patient outcomes. However, the optimal use and place of perampanel in clinical practice has not yet been clearly defined for the Asian patient population. Use of perampanel in clinical practice varies markedly across the Asia region because of variation in knowledge, attitudes, and practice. There is currently no specific guidance on best practices for prescribing perampanel in Asian patients or how to optimize treatment strategies to maximize adherence. A group of epilepsy experts attended a virtual meeting in September 2020 to discuss their experience with using perampanel in the Asian practice setting, including their views regarding appropriate patient populations, optimal starting and maintenance doses, optimal titration regimens, key barriers to adherence, and prevention and management of adverse events. This article summarizes key clinical and realworld evidence for perampanel and consolidates the experts' opinions on optimization of perampanel prescribing and adherence in real-world practice, providing practical strategies for clinicians to implement to improve outcomes for people with epilepsy in Asia.
\end{abstract}

Keywords: perampanel, epilepsy, real-world experience, Asia

\section{Introduction}

Approximately 23 million people in Asia are living with epilepsy, with prevalence varying across countries in the region from 1.5 to 14.0 per 1000 person-years. ${ }^{1}$ Despite treatment with anti-seizure medication (ASM), however, more than onethird of patients still experience seizures that remain uncontrolled. ${ }^{2}$ Drug-refractory epilepsy is associated with reduced quality of life and increased rates of hospitalization, morbidity, and mortality. ${ }^{3}$ In addition, patients whose seizures are poorly controlled face a greater burden of healthcare costs than those whose seizures are in remission. ${ }^{4}$ With each subsequent ASM regimen trialed, the probability of achieving seizure freedom diminishes substantially; most patients who gain seizure control do so with the first or second ASM prescribed. ${ }^{2}$ Therefore, early selection of an effective ASM for initial monotherapy or as early adjunctive therapy is critical for realizing the best possible therapeutic outcomes.

Another key factor contributing to poorly controlled epilepsy is inadequate adherence to ASM therapy. ${ }^{5}$ Non-adherence in epilepsy is estimated to range from $30 \%$ to $50 \%$ of patients, similar to what is observed with other chronic 
illnesses. ${ }^{5}$ Complicated ASM regimens are believed to negatively impact patient adherence, and simplifying treatment strategies by utilizing monotherapy and/or reducing dose frequency can be of benefit. ${ }^{6}$ Prescribing an ASM with characteristics that promote adherence contributes to achieving seizure freedom.

Perampanel is a first-in-class AMPA receptor antagonist approved for the treatment of epilepsy and has broadspectrum efficacy. ${ }^{7-12}$ Perampanel is indicated by the US Food and Drug Administration for treatment of focal onset seizures (FOS), with or without focal-to-bilateral tonicclonic seizures (FBTCS), in patients $\geq 4$ years of age (monotherapy and adjunctive therapy) and as adjunctive therapy in the treatment of primary generalized tonicclonic seizures (GTCS) in patients with epilepsy $\geq 12$ years of age. ${ }^{13}$ In addition, perampanel has a once-daily dosing schedule that supports patient adherence.

Perampanel is already widely indicated for adjunctive use in Asia, and monotherapy is progressively gaining approval across the region, which will foster the earlier use of perampanel to achieve improved patient outcomes. However, there are challenges in the adoption of perampanel in real-world settings in Asia because of variation in knowledge, attitudes, and practice across the region. The optimal use and place of perampanel in clinical practice has not yet been clearly defined for the Asian patient population, and it is therefore at times inaccurately perceived as an agent that should be reserved for later stages of treatment. There is currently no specific guidance on best practices for prescribing perampanel in Asian patients or how to optimize treatment strategies to maximize adherence.

To address these gaps, this paper provides key recommendations on perampanel use from seven epilepsy experts in four Asian countries (China [2], Japan, Taiwan [2], and Thailand [2]) who gathered at a virtual expert advisory board meeting in September 2020. The purpose of the meeting was to discuss the contemporary use of perampanel in clinical practice in Asian countries, share best practice as it relates to prescribing perampanel in Asian patients, identify key barriers to adherence, and develop recommendations that can be easily implemented by clinicians across the region.

This paper describes the consolidated guidance on and insights into optimizing perampanel prescribing and adherence generated at the expert meeting.

\section{Key Clinical and Real-World Evidence on Perampanel}

Based on clinical trial data and real-world experience, perampanel is observed to have broad-spectrum efficacy in a range of seizure types when used as both monotherapy and adjunctive therapy. ${ }^{7-12}$ Clinical studies and real-world evidence have demonstrated the benefits of initiating perampanel at low doses $(\leq 6 \mathrm{mg})^{7-12}$ and utilizing a slow titration strategy (increasing dose by $2 \mathrm{mg}$ at $\geq 2$-week intervals). ${ }^{7,14-16}$ Initiating perampanel at an early stage (as initial monotherapy or early adjunctive therapy) has also been shown to be associated with better patient outcomes; these data are described below.

\section{Clinical Trial Data Monotherapy}

FREEDOM (Study 342) was a Phase III, open-label study in Japan and South Korea of the efficacy and safety of perampanel monotherapy in patients with FOS with or without FBTCS for 26 weeks $(\mathrm{N}=73)$. All patients were newly diagnosed with epilepsy or had experienced seizure recurrence after a period of remission at least 2 years after the cessation of the last ASM treatment. ${ }^{8}$ Patients were treated with perampanel $4 \mathrm{mg} / \mathrm{day}$, which could be titrated to $8 \mathrm{mg}$ /day following a seizure. Perampanel monotherapy was found to be efficacious, with a $63.0 \%$ seizure freedom rate achieved in patients who were maintained on the $4 \mathrm{mg}$ /day dose and $74.0 \%$ overall (last evaluated dose, 4 or $8 \mathrm{mg}$ /day). The safety profile of perampanel $4 \mathrm{mg} /$ day was found to be consistent with that of Phase III studies; treatment-emergent adverse events were reported in $64.0 \%$ of patients in the low-dose cohort and were the cause of withdrawal for only eight patients $(9.0 \%)$.

\section{Early Adjunctive Therapy}

FAME (Study 412) was a Phase IV, open-label study of the efficacy and safety of perampanel as the first adjunctive therapy to monotherapy in patients with FOS with or without FBTCS in Korea $(\mathrm{N}=85){ }^{7}$ Patients received perampanel $4 \mathrm{mg} /$ day, up-titrated by $2 \mathrm{mg}$ over $\geq 2$-week intervals to a maximum of $12 \mathrm{mg} /$ day if required. During the 24-week maintenance period, perampanel was found to be an effective broad-spectrum, first-line adjunctive therapy in patients for whom ASM monotherapy was insufficient. A $50 \%$ responder rate $(\geq 50 \%$ reduction in total seizure frequency) was observed in $80 \%$ of patients during the maintenance period, with seizure improvements seen at both low (4 and $6 \mathrm{mg} /$ day) and high $(8,10$, and $12 \mathrm{mg} /$ 
day) doses. In addition, post hoc analyses suggested that when stratified by titration speed (slow: up-titration performed at $\geq 2$-week intervals; fast: up-titration performed at $<2$-week intervals), the proportions of patients in the slow titration group who achieved $50 \%, 75 \%$, or $100 \%$ responder rates were numerically higher than those in the fast titration group $(83.1 \%$ vs $73.1 \%, 72.9 \%$ vs $69.2 \%$, and $49.2 \%$ vs $42.3 \%$, respectively). Overall, perampanel was well tolerated, with most treatment-emergent adverse events characterized as mild. Furthermore, post hoc analyses demonstrated that when stratified by titration speed, the incidence of treatment-emergent adverse events was $82.9 \%$ for the fast titration group compared with $70.5 \%$ for the slow titration group, suggesting that slower up-titration may be a useful strategy to reduce the overall incidence of adverse events with perampanel.

In a pooled Phase III analysis, efficacy and safety of low-dose (4 mg/day) perampanel as first adjunctive or later adjunctive therapy in patients with FOS with or without FBTCS was assessed $(\mathrm{N}=979) .{ }^{11}$ Compared with placebo, perampanel $4 \mathrm{mg}$ /day conferred significantly greater median percentage reductions in the frequency of all focal seizures per 28 days $(21.1 \%$ vs $12.6 \%, P=0.0004)$. Patients who received perampanel $4 \mathrm{mg}$ /day also experienced a significant reduction in FBTC seizures compared with placebo $(49.8 \%$ vs $17.4 \%, P=0.0001)$. Seizure freedom rates were also significantly higher with perampanel $4 \mathrm{mg} /$ day than with placebo for all focal seizures $(3.6 \% \mathrm{vs}$ $0.8 \%, P=0.0018)$ and for FBTCS $(18.7 \%$ vs $11.1 \%$, $P=0.0424)$. The rate of reported adverse events with adjunctive perampanel $4 \mathrm{mg} /$ day in patients with focal seizures was $30.5 \%$ overall, with the most frequently reported events being dizziness (7.3\%), somnolence $(3.6 \%)$, and headache $(3.1 \%)$. Treatment-emergent adverse events led to discontinuation in only $2 \%$ of patients receiving perampanel $4 \mathrm{mg} /$ day.

Study 311 was a global, multicenter, open-label, singlearm study that evaluated the safety and efficacy of perampanel in children aged 4 to $<12$ years of age with FOS or GTCS $(\mathrm{N}=180) .{ }^{17}$ Perampanel was demonstrated to be well tolerated, with safety and efficacy outcomes consistent with those seen in adult studies.

\section{Real-World Data Monotherapy}

A retrospective, multicenter, registry study assessed the efficacy and tolerability of perampanel for routine clinical treatment of FOS and GTCS in Spain $(\mathrm{N}=98)$. Patients were initiated on primary perampanel monotherapy or switched to monotherapy by removal of all concomitant ASMs and treated for a median of 14 months (range: 157). The median maintenance dose of perampanel used was $4 \mathrm{mg}$ /day (interquartile range: $4-6) .^{10}$ At 3, 6, and 12 months since initiation of perampanel treatment, respectively, retention rates were $93.8 \%, 89.3 \%$, and $80.9 \%$, responder rates were $79.6 \%, 70.1 \%$, and $52.8 \%$, and seizure freedom rates were $62.7 \%, 56.1 \%$, and $41.5 \%$. Regardless of whether perampanel monotherapy was given as the primary or secondary treatment, retention rates did not vary $(P=0.57)$. While not reaching statistical significance, seizure freedom rates during the whole observation period were higher in the primary monotherapy group than in the converted monotherapy group $(71.4 \%$ vs $51.2 \%$ at 6 months and $58.3 \%$ vs $37.5 \%$ at 12 months). Perampanel monotherapy was generally well tolerated, with most reported adverse events being mild in nature.

A retrospective, multicenter, non-interventional study evaluated patient retention on perampanel as primary or secondary monotherapy for FOS at specialist epilepsy centers in Russia and Europe $(\mathrm{N}=60) .{ }^{9}$ The primary monotherapy cohort received perampanel in the absence of any other ASM, and the secondary monotherapy group were converted from adjunctive therapy by withdrawing all concomitant ASMs. Overall, perampanel retention rates at 3 and 6 months were $95 \%$ and $74 \%$, respectively. At study cut-off at 24 months, $68 \%$ of patients were continuing to take perampanel treatment as primary or secondary monotherapy.

\section{Early Adjunctive Therapy}

A prospective, observational study investigated the efficacy and safety of perampanel as early adjunctive treatment in patients with non-controlled FOS with or without FBTCS in Spain $(\mathrm{N}=113) .^{3}$ The median dose of perampanel prescribed was $6 \mathrm{mg} /$ day (range: 4-12). Patients achieved significantly higher rates of seizure freedom at 12 months when perampanel was initiated as a first versus second adjunctive treatment $(38.1 \%$ vs $19.7 \%$, respectively; $P=0.033$ ). The treatment retention rate was high, with $83.2 \%$ and $80.5 \%$ of patients still receiving perampanel at 6 and 12 months, respectively. Overall, treatment-emergent adverse events were reported in $30.1 \%(34 / 113)$ of patients, and psychiatric adverse events occurred in $20.4 \%(23 / 113)$; most events were mild to moderate in severity. The percentage of patients who experienced adverse events $(36.6 \%$ vs $19 \%, P=0.049)$ 
and psychiatric adverse events $(28.2 \%$ vs $7.1 \%, P=0.008)$ was significantly higher when perampanel was added as second adjunctive treatment than when it was used as first adjunctive treatment.

The FYDATA study was a retrospective, multicenter, observational study investigating the safety and efficacy of perampanel as an early adjunctive in patients with FOS $(\mathrm{N}=464) .{ }^{14}$ A large proportion of patients were highly treatment refractory, with an average (range) of 7.8 (118) prior and $2.8(1-5)$ concurrent ASMs prescribed. At 1 year, the perampanel retention rate was $60.6 \%$. Seizure reduction, safety, and tolerability were similar to those seen in clinical trials. The effect of titration schedule was also investigated; significantly fewer patients on slow titration schemes ( $2 \mathrm{mg}$ increase every 3-4 weeks) experienced treatment-emergent adverse events than patients on fast titration schemes (2 $\mathrm{mg}$ increase every week; $P=0.006)$. In addition, fewer patients on slow titration schemes experienced psychiatric adverse events compared with patients on fast titration, although this was not significant $(P=0.152)$.

A retrospective, observational, two-center study analyzed the efficacy of perampanel as early adjunctive treatment in patients with FOS in Japan $(\mathrm{N}=51) .{ }^{18}$ The seizure-freedom rates at 6 and 12 months were $29 \%$ and $28 \%$, respectively. In the 30 patients who received perampanel continuously for 6 months, the seizure-freedom rates were significantly higher in those who used perampanel as a first adjunctive treatment (75\%) compared with those who used it later (31.8\%). The most frequently prescribed perampanel dose among patients who achieved seizure freedom was $2 \mathrm{mg} /$ day.

A multicenter, retrospective, observational study evaluated the efficacy and safety of perampanel as early adjunctive treatment in patients with idiopathic generalized epilepsy (IGE), including myoclonic and absence seizures. ${ }^{12}$ The median dose of perampanel prescribed was $6 \mathrm{mg} /$ day. Rates of retention and seizure freedom were significantly higher when perampanel was used as early ( $\leq 2$ prior ASMs) versus late $(>2$ prior ASMs) adjunctive therapy.

The effect of perampanel in the treatment of myoclonic and absence seizures was also evaluated in a post hoc analysis of a Phase III study ( $\mathrm{N}=163) .{ }^{19}$ Myoclonic seizure freedom was achieved in $16.7 \%$ of perampanel-treated patients compared with $13.0 \%$ of those treated with placebo, and absence seizure freedom was achieved in $22.2 \%$ versus $12.1 \%$, respectively ( $P$ values not reported).
A multicenter, retrospective, observational study determined the 12-month efficacy and tolerability of perampanel as an adjunctive treatment in children $\geq 4$ years of age with epilepsy in Korea $(\mathrm{N}=220) .{ }^{20}$ Response and seizure freedom rates were $43.6 \%$ and $17.7 \%$, respectively, with no significant differences across age groups. Adverse events were reported in $40 \%$ of patients, but most were of mild severity.

\section{Special Populations}

A retrospective, observational study evaluated the efficacy and safety of perampanel as an adjunctive treatment in patients with refractory status epilepticus (SE) in Taiwan $(\mathrm{N}=81) .{ }^{21}$ Clinical improvement and EEG resolution $\leq 72$ hours after perampanel administration was achieved in 27 (33.3\%) patients, with a median response time of 40 hours (1-69 hours). The median dose of perampanel initially prescribed was $4 \mathrm{mg} /$ day (2-36 mg/day). Perampanel was well tolerated with no cardiorespiratory events or laboratory changes recorded. Larger clinical studies are required to fully assess the value of perampanel for status epilepticus. ${ }^{22}$

Perampanel has shown efficacy and safety in elderly patients with epilepsy; the once-daily dosing schedule has been highlighted as a particular advantage in this cohort. ${ }^{23}$ As with younger patients, utilizing a slow titration approach and a low starting dose help to minimize adverse events.

There are currently only preliminary data regarding the use of perampanel in women with epilepsy; further outcome data are required to estimate the prevalence of adverse pregnancy outcomes with perampanel exposure. ${ }^{24}$

\section{Best Practice Expert Recommendations Initiation of Perampanel in Asian Populations}

Consistent with the published data, the expert group recommend early initiation of perampanel as initial monotherapy or early adjunctive therapy. In their clinical experience, they have found that patients tend to achieve better outcomes with early use than when perampanel is initiated later in their treatment journey.

The following patient groups were also highlighted by the expert group as those who could most benefit from treatment with perampanel in the following settings.

\section{Perampanel as Initial Monotherapy}

- FOS with or without FBTCS in adolescents $(>12$ years of age), adults, and the elderly. ${ }^{8}$ 
Perampanel is also likely to be of particular benefit when used as initial monotherapy in the following specific groups of patients with epilepsy; however, further studies are needed to fully evaluate its effectiveness in these settings:

- FOS or generalized onset seizures (GOS) in pediatric patients

- Myoclonic epilepsy (eg progressive myoclonic epilepsy [PME], juvenile myoclonic epilepsy)

\section{Perampanel as Early Adjunctive Therapy}

Perampanel is likely to be of particular benefit when used as early adjunctive therapy in the following specific groups of patients with epilepsy; however, further studies are needed to fully evaluate its effectiveness in these settings:

- Patients with focal lesions ${ }^{25}$ (eg focal cortical dysplasia, tuberous sclerosis complex)

- Tonic-clonic seizures in $\mathrm{IGE}^{12}$

- $\mathrm{PME}^{26}$

- Brain-tumor-related epilepsy ${ }^{27}$

\section{Dose Optimization in Asian Populations Starting Dose}

The expert group advise that the optimal perampanel starting dose in Asian patients is $2 \mathrm{mg}$ /day, consistent with the approved prescribing information. However, if adverse events are observed at the $2 \mathrm{mg}$ /day starting dose and perampanel suspension/granule formulations are available, a reduction to a $1 \mathrm{mg}$ /day starting dose may be considered.

\section{Titration Strategy}

The expert group recommend following a slow titration strategy for perampanel in Asian patients by increasing the daily dose by $2 \mathrm{mg}$ every 2 weeks or at even longer intervals. Where perampanel suspension/granule formulations are available, an alternative strategy is to increase the perampanel dose by $1 \mathrm{mg}$ every 2 weeks.

The expert group suggest that rapid titration (increasing the dose more frequently than every 2 weeks) may be considered in patients with concomitant enzyme-inducing ASMs, status epilepticus and other emergencies, severe epilepsy, frequent seizures, or brain-tumor-related epilepsy.

\section{Maintenance Dose}

Based on their clinical experience, the expert group recommend that the optimal maintenance dose for perampanel for most patients is either $4 \mathrm{mg} /$ day or $6 \mathrm{mg} /$ day; these doses offer effective seizure reduction while minimizing adverse events in most patients. In some cases, a maintenance dose of $8 \mathrm{mg} /$ day or higher may be required for optimal effect, but this is not a frequent occurrence in the experience of the expert group.

\section{Key Considerations When Initiating Perampanel in Asian Populations Age}

ASM options are limited in elderly patients because of safety concerns, but perampanel, particularly when administered early, has a favorable efficacy and safety profile in the experience of the expert group.

The group recommend consideration of a lower starting dose ( $\leq 1 \mathrm{mg} /$ day) for elderly and pediatric populations, with slow up-titration of perampanel dose at $>2$-week intervals when feasible.

\section{Comorbidities}

The expert group advise that treatment with perampanel may offer specific benefits to patients with certain comorbidities.

Owing to its neutral effect on cognitive function, ${ }^{28}$ perampanel treatment may be advantageous for patients with existing mild cognitive abnormalities. Cognitive impairment such as memory loss, mental slowness, and attention deficits are commonly associated with epilepsy. ${ }^{29}$ Approximately a quarter of people with epilepsy have an intellectual disability, ${ }^{30}$ and these impairments can significantly impact patients' quality of life. ${ }^{3}$ Cognitive impairment can also be an adverse effect associated with ASM, so early management is important if this does occur. ${ }^{29}$ Based on the experts' clinical experience, perampanel appears to exert no negative impact on cognitive function and therefore is a rational option for these patients.

Another group of patients identified as likely to benefit markedly from treatment with perampanel are those who suffer from insomnia. Problems with sleep are common in people with epilepsy, with prevalence estimates ranging from $24 \%$ to $55 \%{ }^{31}$ Insomnia exerts a negative effect on seizure control and quality of life in this patient group. ${ }^{31}$ The expert group advise that perampanel may contribute to improved sleep quality, which would be highly advantageous for these patients.

\section{Concomitant Medications}

Enzyme-inducing ASMs have been shown to promote the metabolism of concomitant ASMs, which may result in serum concentrations of the ASM being reduced and 
seizure control being adversely affected. ${ }^{32}$ Given its extensive metabolism, systemic exposure to perampanel may be reduced when administered in conjunction with an enzyme-inducing ASM. ${ }^{33}$

In the experts' experience, a higher maintenance dose ( $>6 \mathrm{mg} /$ day) of perampanel may be required in cases where patients are taking concomitant enzyme-inducing ASMs. Utilizing a more rapid titration approach may also be of benefit.

\section{Optimizing Perampanel Adherence in Asian Populations}

ASM non-adherence is associated with a wide range of negative consequences. ${ }^{5,6,34}$ It is one of the key contributors to uncontrolled seizures and has been linked with a $>3$-fold increased risk of mortality compared with patients who are adherent to therapy. ${ }^{6}$ ASM nonadherence has also been correlated with higher rates of hospital admissions, increased healthcare costs, and reduced quality of life. ${ }^{6}$ However, as patients are often unaware of the serious consequences of non-adherence, they may choose to discontinue treatment. ${ }^{29}$

There are multiple barriers to good ASM adherence and long-term treatment retention, including frequent ASM dosing schedules and treatment-emergent adverse events; addressing these factors is therefore critical for optimal perampanel adherence and treatment outcomes. ${ }^{34}$ Newer ASMs such as perampanel tend to have a more favorable safety profile than first-generation ASMs ${ }^{29}$ however, early and frequent follow-up after initiation of perampanel is critical to identify and effectively manage adverse events before they result in patient discontinuation.

The following types of adverse events are highlighted by the expert group as being particularly detrimental to adherence to perampanel in the Asian patient population: behavioral and psychiatric adverse events (eg aggression, irritability, depression); somnolence and dizziness; and ataxia/gait disturbance.

Owing to its long half-life, perampanel requires only once-daily dosing, which has been demonstrated to be an effective strategy for improving patient adherence and is also more forgiving in the event of missed doses. ${ }^{35}$ The expert group recommend instructing patients to schedule their daily perampanel dose for the evening and to go directly to bed afterwards.

The expert group advise the following approaches when prescribing perampanel to prevent and manage adverse events and maximize adherence and long-term retention on treatment.

\section{Strategies for the Prevention of Adverse Events with Perampanel in Asian Patients}

- At perampanel initiation, patients and/or family members should be advised on the criticality of adherence in relation to the benefits of treatment. Clinicians should also educate patients and/or family members about potential adverse effects so that they are not surprised or overly distressed should they occur and know to report them to their treating clinician as soon as practicable

- Inform patients and carers on how adverse events can be prevented and provide reassurance that they can be effectively managed if they do arise and do not necessarily mean that the patient will discontinue perampanel

- Initiate perampanel at a low dose and titrate to the maintenance dose slowly

- Inform patients and carers that adverse events frequently occur in the early phase of treatment, and patients become better able to tolerate these effects the longer they take perampanel

- Instruct patients or carers to take/administer perampanel at night, just before retiring to bed. This is a critical step to help minimize the impact of somnolence, and dizziness in particular, which are adverse events that may result in discontinuation

- Proactively monitor patients for the development of any adverse events during treatment. Ensure that the patient schedules their first follow-up appointment within 1 week of perampanel initiation, then every 2-4 weeks thereafter, depending on the patient's individual circumstances and availability

- During the titration period, clinicians must pay particular attention to emergent adverse events as it is the period of highest risk. Greater scrutiny should also be applied when a higher dose of perampanel has been prescribed for treatment maintenance

- In adolescent patients or patients with a history of any psychiatric comorbidity or family history of psychiatric disorders, prescribers may consider utilizing a slow dose-titration strategy and more intensive monitoring

\section{Strategies for Managing Adverse Events with Perampanel in Asian Patients}

Should adverse effects develop while the patient is taking perampanel, the expert group recommend the following strategies: 
- The patient should not discontinue treatment abruptly but should consult their treating clinician for advice

- If the adverse effect develops during the titration period, slow the titration speed by increasing the time between dose increases to 4-week intervals. Alternatively, down-titrate to the previous tolerated dosage for a 2- to 4-week period before attempting another slow up-titration

- If the adverse effect develops during the maintenance period, then reduce the perampanel dose for a short period of time until the adverse effect resolves; the dose may be up-titrated again once the patient is better tolerating the medication

- Reiterate the importance of taking perampanel immediately before going to bed should somnolence or dizziness occur

\section{Conclusions}

Perampanel offers broad-spectrum efficacy in a range of seizure types as a once-daily dose; however, clinical data in Asian patients are limited and practical guidance has been lacking on how to optimize the use of this agent in the Asian setting. This expert group recommend several practical strategies that clinicians in the region can easily incorporate into their clinical practice when initiating perampanel for the treatment of epilepsy. Perampanel is likely to be effective in FOS with or without FBTCS across a wide range of age groups, in GOS, and in myoclonic epilepsy. Initiating perampanel early in the treatment journey can optimize efficacy. Selecting a low starting dose and utilizing a slow titration strategy is recommended to minimize the impact of adverse effects, maximize adherence, and increase patient retention. Educating patients and carers about treatment-emergent adverse events that frequently develop in the early phases of treatment is advised, as is instructing patients to take their dose at bedtime to maximize tolerance.

\section{Acknowledgments}

Writing assistance was provided by Leonie Brown from AMICULUM, funded by Eisai Co, Ltd.

\section{Author Contributions}

All authors made a significant contribution to the work reported, whether that is in the conception, study design, execution, acquisition of data, analysis, and interpretation, or in all these areas; took part in drafting, revising or critically reviewing the article; gave final approval of the version to be published; have agreed on the journal to which the article has been submitted; and agreed to be accountable for all aspects of the work. Yotin Chinvarun is the lead author.

\section{Funding}

The authors disclosed receipt of the following financial support for the authorship and/or publication of this article: this work was supported by Eisai Co, Ltd, Tokyo, Japan.

\section{Disclosure}

Takamichi Yamamoto has received speaker's honoraria from Daiichi-Sankyo, Eisai, UCB Pharma, LivaNova, PLC, and Otsuka Pharmaceutical, participated in advisory boards for Eisai and served as Sponsor's Responsible Medical Officer in the FREEDOM Trial (Study 342). Yotin Chinvarun, ChinWei Huang, Hsiu-Fen Lee, Ye Wu, Jing Ding, and Surachai Likasitwattanakul declare no competing interests.

\section{References}

1. Trinka E, Kwan P, Lee B, Dash A. Epilepsy in Asia: disease burden, management barriers, and challenges. Epilepsia. 2019;60(S1):7-21. doi:10.1111/epi.14458

2. Chen Z, Brodie MJ, Liew D, Kwan P. Treatment outcomes in patients with newly diagnosed epilepsy treated with established and new antiepileptic drugs: a 30-year longitudinal cohort study. JAMA Neurol. 2018;75(3):279-286. doi:10.1001/jamaneurol.2017.3949

3. Abril Jaramillo J, Estevez Maria JC, Giron Ubeda JM, et al. Effectiveness and safety of perampanel as early add-on treatment in patients with epilepsy and focal seizures in the routine clinical practice: Spain Prospective Study (PERADON). Epilepsy Behav. 2020;102:106655. doi:10.1016/j.yebeh.2019.106655

4. Gao L, Xia L, Pan SQ, Xiong T, Li SC. Burden of epilepsy: a prevalence-based cost of illness study of direct, indirect and intangible costs for epilepsy. Epilepsy Res. 2015;110:146-156. doi:10.1016/j. eplepsyres.2014.12.001

5. Jones RM, Butler JA, Thomas VA, Peveler RC, Prevett M. Adherence to treatment in patients with epilepsy: associations with seizure control and illness beliefs. Seizure. 2006;15(7):504-508. doi:10.1016/j. seizure.2006.06.003

6. Malek N, Heath CA, Greene J. A review of medication adherence in people with epilepsy. Acta Neurol Scand. 2017;135(5):507-515. doi:10.1111/ane.12703

7. Kim JH, Kim DW, Lee SK, et al. First add-on perampanel for focal-onset seizures: an open-label, prospective study. Acta Neurol Scand. 2020;141(2):132-140. doi:10.1111/ane.13197

8. Yamamoto T, Lim SC, Ninomiya H, et al. Efficacy and safety of perampanel monotherapy in patients with focal-onset seizures with newly diagnosed epilepsy or recurrence of epilepsy after a period of remission: the Open-Label Study 342 (FREEDOM Study). Epilepsia Open. 2020;5(2):274-284. doi:10.1002/epi4.12398

9. Gil-Nagel A, Burd S, Toledo $\mathrm{M}$, et al. A retrospective, multicentre study of perampanel given as monotherapy in routine clinical care in people with epilepsy. Seizure. 2018;54:61-66. doi:10.1016/j.seizure. 2017.10.015 
10. Toledano Delgado R, García-Morales I, Parejo-Carbonell B, et al. Effectiveness and safety of perampanel monotherapy for focal and generalized tonic-clonic seizures: experience from a national multicenter registry. Epilepsia. 2020;61(6):1109-1119. doi:10.1111/epi. 16548

11. Steinhoff BJ, Patten A, Williams B, Malhotra M. Efficacy and safety of adjunctive perampanel $4 \mathrm{mg} / \mathrm{d}$ for the treatment of focal seizures: a pooled post hoc analysis of four randomized, double-blind, phase III studies. Epilepsia. 2020;61(2):278-286. doi:10.1111/epi.16428

12. Villanueva V, Montoya J, Castillo A, et al. Perampanel in routine clinical use in idiopathic generalized epilepsy: the 12-month GENERAL Study. Epilepsia. 2018;59(9):1740-1752. doi:10.1111/ epi. 14522

13. Fycompa prescribing information; 2020. Available from: https:// www.fycompa.com/-/media/Files/Fycompa/Fycompa_Prescribing_ Information.pdf. Accessed September 11, 2020.

14. Villanueva V, Garces M, Lopez-Gonzalez FJ, et al. Safety, efficacy and outcome-related factors of perampanel over 12 months in a real-world setting: the FYDATA Study. Epilepsy Res. 2016;126:201-210. doi:10.1016/j.eplepsyres.2016.08.001

15. Basheikh M, Sadler RM. Retention rate and efficacy of perampanel with a slow titration schedule in adults. Can $J$ Neurol Sci. 2020;48:1-7.

16. Shah E, Reuber M, Goulding P, Flynn C, Delanty N, Kemp S. Clinical experience with adjunctive perampanel in adult patients with uncontrolled epilepsy: a UK and Ireland multicentre study. Seizure. 2016;34:1-5. doi:10.1016/j.seizure.2015.10.017

17. Fogarasi A, Flamini R, Milh M, et al. Open-label study to investigate the safety and efficacy of adjunctive perampanel in pediatric patients ( 4 to $<2$ years) with inadequately controlled focal seizures or generalized tonic-clonic seizures. Epilepsia. 2020;61(1):125-137. doi:10. 1111/epi.16413

18. Takahashi S, Shimizu K, Inaji M, Hashimoto S, Yamamoto S, Maehara T. Effectiveness of perampanel as a first add-on antiepileptic drug for the treatment of partial epilepsy. Epilepsy Behav. 2019;100 (Pt A):106492. doi:10.1016/j.yebeh.2019.106492

19. Brandt C, Wechsler RT, O'Brien TJ, et al. Adjunctive perampanel and myoclonic and absence seizures: post hoc analysis of data from study 332 in patients with idiopathic generalized epilepsy. Seizure. 2020;80:115-123. doi:10.1016/j.seizure.2020.06.011

20. Hwang SK, Lee YJ, Nam SO, et al. Real-life effectiveness and tolerability of perampanel in pediatric patients aged 4 years or older with epilepsy: a Korean national multicenter study. J Clin Neurol. 2020;16(1):53-59. doi:10.3988/jen.2020.16.1.53

21. Lim SN, Wu T, Tseng WJ, et al. Efficacy and safety of perampanel in refractory and super-refractory status epilepticus: cohort study of 81 patients and literature review. J Neurol. 2021. doi:10.1007/s00415021-10506-9

22. Brigo F, Lattanzi S, Rohracher A, et al. Perampanel in the treatment of status epilepticus: a systematic review of the literature. Epilepsy Behav. 2018;86:179-186. doi:10.1016/j.yebeh.2018.07.004
23. Rohracher A, Kalss G, Kuchukhidze G, et al. New anti-seizure medication for elderly epilepsy patients - a critical narrative review. Expert Opin Pharmacother. 2021;22(5):621-634. doi:10.1080/ 14656566.2020.1843636

24. Vazquez B, Tomson T, Dobrinsky C, Schuck E, O’Brien TJ. Perampanel and pregnancy. Epilepsia. 2021;62(3):698-708. doi:10. 1111/epi.16821

25. Nilo A, Pauletto G, Gigli GL, Vogrig A, Dolso P, Valente M. Perampanel as add-on therapy in epilepsies with known etiology: a single center experience with long-term follow-up. Epilepsy Behav Rep. 2021;15:100393. doi:10.1016/j.ebr.2020.100393

26. Ferlazzo E, Trenite DK, Haan GJ, et al. Update on pharmacological treatment of progressive myoclonus epilepsies. Curr Pharm Des. 2017;23(37):5662-5666. doi:10.2174/1381612823666170809114654

27. Coppola A, Zarabla A, Maialetti A, et al. Perampanel confirms to be effective and well-tolerated as an add-on treatment in patients with brain tumor-related epilepsy (PERADET Study). Front Neurol. 2020;11:592. doi:10.3389/fneur.2020.00592

28. Rea R, Traini E, Renna R, Pagliuca F, Pezzella M, Pagliuca M. Efficacy and impact on cognitive functions and quality of life of perampanel as first add-on therapy in patients with epilepsy: a retrospective study. Epilepsy Behav. 2019;98(Pt A):139-144. doi:10.1016/j.yebeh.2019.07.005

29. Trinka E, Steinhoff BJ, Nikanorova M, Brodie MJ. Perampanel for focal epilepsy: insights from early clinical experience. Acta Neurol Scand. 2016;133(3):160-172. doi:10.1111/ane.12529

30. Carreňo M, Trinka E. Reflections on the use of perampanel in epilepsy - lessons from the clinic and real-world evidence. Eur Neurol Rev. 2017;12(1):17-23.

31. Quigg M, Gharai S, Ruland J, et al. Insomnia in epilepsy is associated with continuing seizures and worse quality of life. Epilepsy Res. 2016;122:91-96. doi:10.1016/j.eplepsyres.2016.02.014

32. Johannessen SI, Landmark CJ. Antiepileptic drug interactions - principles and clinical implications. Curr Neuropharmacol. 2010;8 (3):254-267. doi:10.2174/157015910792246254

33. Gidal BE, Laurenza A, Hussein Z, et al. Perampanel efficacy and tolerability with enzyme-inducing AEDs in patients with epilepsy. Neurology. 2015;84(19):1972-1980. doi:10.1212/WNL.00000000000 01558

34. O' Rourke G, O' Brien JJ. Identifying the barriers to antiepileptic drug adherence among adults with epilepsy. Seizure. 2017; 45:160-168. doi:10.1016/j.seizure.2016.12.006

35. Gidal BE, Majid O, Ferry J, et al. The practical impact of altered dosing on perampanel plasma concentrations: pharmacokinetic modeling from clinical studies. Epilepsy Behav. 2014;35:6-12. doi:10. 1016/j.yebeh.2014.03.013
Therapeutics and Clinical Risk Management

\section{Publish your work in this journal}

Therapeutics and Clinical Risk Management is an international, peerreviewed journal of clinical therapeutics and risk management, focusing on concise rapid reporting of clinical studies in all therapeutic areas, outcomes, safety, and programs for the effective, safe, and sustained use of medicines. This journal is indexed on PubMed Central, CAS,
EMBase, Scopus and the Elsevier Bibliographic databases. The manuscript management system is completely online and includes a very quick and fair peer-review system, which is all easy to use. Visit http://www.dovepress.com/testimonials.php to read real quotes from published authors. 